'Breeding for Success: Diversity in Action' C.F. Mercer (ed).

Proceedings of the $13^{\text {th }}$ Australasian Plant Breeding Conference,

Christchurch, New Zealand 18-21 April 2006. pp. 1184-1191.

\title{
Increased cuticular wax accumulation and enhanced drought tolerance in transgenic alfalfa (Medicago sativa) by overexpression of a transcription factor gene
}

\section{Ji-Yi Zhang, Mary Sledge, Joseph Bouton and Zeng-Yu Wang}

Forage Improvement Division, The Samuel Roberts Noble Foundation, Ardmore, OK

73401, USA

Email: zywang@noble.org

Abstract. Plant cuticular waxes play an important role in protecting aerial organs from damage caused by multiple environmental stresses such as drought, cold, UV radiation, pathogen infection, and insect attack. We characterized a novel AP2 domain-containing transcription factor gene, designated WXP1, from the model legume plant Medicago truncatula. The gene is able to activate wax production and confer drought tolerance in alfalfa (Medicago sativa). The predicted protein of WXP1 has 371 aa; it is one of the longest peptides of all the single AP2 domain proteins in $M$. truncatula. Transcript level of WXP1 is inducible by cold, ABA and drought treatment in shoot tissues.

Overexpression of WXP1 under the control of CaMV35S promoter led to a significant increase in cuticular wax loading on leaves of transgenic alfalfa. Transgenic leaves showed reduced water loss and chlorophyll leaching. Transgenic alfalfa plants with increased cuticular waxes showed enhanced drought tolerance demonstrated by delayed wilting after watering was ceased and quicker and better recovery when the dehydrated plants were re-watered.

\section{Introduction}

Cuticle is one of the most important barriers for all terrestrial plants to adapt their above ground tissues to drought, as well as other biotic and abiotic stresses (Jenks and Ashworth 1999). The important physiological and ecological properties of cuticle are related to the presence of cuticular waxes that are embedded within and encase the cutin matrix, in which the cuticle is constructed (Kunst and Samuels 2003, Naâ et al. 1998). Cuticular waxes are complex mixtures of fatty acids, alkanes, primary and/or secondary alcohols, aldehydes, ketones, esters, which all have carbon chain length of 18-35. Some other second metabolites, such as triterpenes, sterols, and flavonoids, are also found sometimes.

Plant cuticular wax biosynthesis and its loading to the plant surface is a complicated but actively regulated process (Jenks et al. 2002, Kunst 2003). Mutant analysis has greatly contributed to the identification of the components and genes involved in wax deposition. However, only limited information is available on the effects of overexpression of these genes in the wild-type background. Overexpression of the condensing enzyme gene CER6/CUT1 under the control of CaMV35S promoter failed to promote wax deposition (Millar et al. 1999), whilst under the control of epidermis-specific CER6 promoter, CER6/CUT1 overexpression led to increased wax load in stems of Arabidopsis (Hooker et al. 2002). The only reports on increased wax accumulation in leaf tissues were by the overexpression of a transcription factor gene (WIN1/SHN1) in Arabidopsis (Aharoni et al. 2004, Broun et al. 2004).

Because multiple biochemical processes are involved in wax biosynthesis, transgenic expression of regulatory genes could be an effective approach to manipulate wax accumulation in plants (Zhang et al. 2005). Most of the transcription factors are 
grouped into gene families according to their well-conserved DNA-binding domains. APETALA 2 (AP2)/Ethylene-responsive element binding factors (ERF or EREBP) domain-containing transcription factor is a group of transcriptional regulators that are specifically found in plants (Okamuro et al. 1997, Riechmann et al. 2000). The AP2 domains in these proteins play a major role in specific promoter DNA sequence/element binding and transcriptional activation. In the past several years, a group of AP2 domaincontaining transcription factors-dehydration-response element binding protein (DREB)/C-repeat binding factor (CBF) genes have been identified and characterized (Novillo et al. 2004, Shinozaki et al. 2003). They are mainly involved in the regulation of abiotic stress inducible genes; overexpression of some members from this subfamily in transgenic Arabidopsis induced a host of genes and conferred stress tolerance (Gilmour et al. 2000, Haake et al. 2002, Jaglo-Ottosen et al. 1998, Kasuga et al. 1999, Stockinger et al. 1997).

Medicago truncatula, barrel medic, is a forage crop that has been developed into a model legume in view of its small diploid genome, self-fertile nature and relatively short life cycle (Cook 1999, Young et al. 2005b). A large number of ESTs $(>226,000)$ have been sequenced from $M$. truncatula and its genespace sequencing is in progress (Young et al. 2005a). We characterized a novel gene from $M$. truncatula, designated WXP1, which activates wax production in the acyl-reduction pathway (Zhang et al. 2005). Overexpression of WXP1 under the control of CaMV35S promoter led to increased cuticular wax loading on leaf surface and enhanced drought tolerance in transgenic alfalfa.

\section{Materials and methods}

Construction of transgene and alfalfa transformation

The coding sequence of WXP1 from Medicago truncatula (genotype Jemalong A17) was PCR amplified using primers 5'-GGTACCATGGATTTCTTCAACA-3' (forward) and 5'-AACCGGTCACCAAATTCATCCA-3' (reverse) and digested by restriction endonuclease Nco I and BstE II. The fragment was inserted into pCAMBIA 3301 vector by replacing the gusA gene, which resulted in a binary vector pC35S-WXP1. An alfalfa genotype, Regen SY-4D, was used for Agrobacterium-mediated transformation to generate transgenic plants. Vegetatively propagated plants from the original Regen SY$4 \mathrm{D}$ clone were used as wild type controls. Alfalfa lines transformed with the original pCAMBIA3301 vector were used as vector controls.

Growth and treatments of alfalfa

The transgenic (T0) or control alfalfa plants were propagated using shoot cuttings. For the analysis of wax content, chlorophyll leaching and leaf water loss rate, samples were taken from plants with 6-7 fully expanded trifoliates on the major stem and 4-6 trifoliates on the branches. All the plants were grown in a greenhouse at $23 / 19^{\circ} \mathrm{C}$ with $14 \mathrm{~h} / 10 \mathrm{~h}$ photoperiod and relative humidity at $\sim 50 \%$. For drought tolerance study, seedlings were transplanted to 4.5 -inch pots and grown in growth chamber at $23 / 19^{\circ} \mathrm{C}$ with $16 \mathrm{~h} / 8 \mathrm{~h}$ photoperiod and relative humidity at $60 \%$. After 20 days of growth, plants (in three replicates) were drought stressed by stopping watering. At the same time, humidity was re-set to $20 \%$ and photoperiod was re-set to $8 \mathrm{~h} / 16 \mathrm{~h}$ in order to keep the stomata closed most of the time. Drought tolerance phenotype was recorded 3-4 days later. When the control plants became totally dried or dead-like ( $\sim 10$ days after watering was withheld), all the pots were re-watered and humidity was re-set to $60 \%$. Plant recovery was scored 2 weeks later. In the greenhouse experiment, the plants were 
subjected to three cycles of drought-recovery treatment. During each cycle of droughtrecovery treatment, plants were drought stressed for 7 days and watering resumed for 2 days.

\section{GC-MS analysis of cuticular wax composition}

Leaf cuticular wax samples were collected from top four fully expanded trifoliates excised from the major stems. The stem cuticular wax samples were collected from the top four internodes. One leaflet was excised from each trifoliate and the four leaflets were combined as one leaf sample. Four internodes were combined as one stem sample. Each sample was inserted into a $20-\mathrm{mL}$ glass tube, and $10-\mathrm{mL}$ (for leaves) or 5-mL (for stem) of hexane (Sigma-Aldrich, Inc., St. Louis, MO) was added. Tissues were agitated for $2 \mathrm{~min}$ on a rotator at $50 \mathrm{rpm}$, and the solvent was decanted into new glass tubes. Tissues and tubes were given a 10-s rinse with the same amount of hexane, and the solution was combined in the new tubes. The hexane-soluble wax extracts were evaporated to a small volume $(\sim 1 \mathrm{~mL})$ under a nitrogen stream and then transferred into 2-mL auto-sampler vials. After complete evaporation in the 2-mL vials, the extracts were resuspended/derivatized in $15.0 \mu \mathrm{L}$ of $70 \%$ Pyridine and 30\% MSTFA (with 0.01 $\mu \mathrm{g} / \mu \mathrm{l}$ of cholesterol as internal standard) for every one $\mathrm{cm}^{2}$ leaf section or one $\mathrm{cm}^{2}$ stem surface area. Derivatization was performed for $60 \mathrm{~min}$ at $50^{\circ} \mathrm{C}$. One microliter of the solution was injected onto an Agilent 6890 gas chromatograph in splitless mode. The injector was held at $280^{\circ} \mathrm{C}$, the oven programmed from $120^{\circ} \mathrm{C}(2 \mathrm{~min})$ to $315^{\circ} \mathrm{C}(8 \mathrm{~min})$ at $5^{\circ} \mathrm{C} / \mathrm{min}$, and the transfer arm held at $250^{\circ} \mathrm{C}$. The GC was coupled to an Agilent 5973 MSD using electron impact ionization with scanning masses 50-650. Duplicate injections were performed for each sample, and the average value of the two injections was used for statistical analysis. Quantification was based on peak areas and the variation in MS sensitivity was adjusted based on the internal standard as described by Jenks et al (1995) and Bergman et al (1991) with modifications. The amount of each cuticular wax component and total wax composition was expressed per unit of leaf surface area. Leaf areas were determined using computer digitization of scanned images. All values represent averages of six replicate plant samples \pm SD.

Quantification of epidermal traits

To quantify leaf water loss rate, the top- $2^{\text {nd }}$ and top- $3^{\text {rd }}$ trifoliates were detached from 20-d-old alfalfa after the plants were kept in constant dark for 10 hours. Dehydration and measurement was performed in a dark room at $23^{\circ} \mathrm{C}$. Humidity was around $50 \%$ in the room.

\section{Results}

Sequence Comparison and Transgenic Expression of WXP1 in alfalfa

About 80 AP2 domain-containing transcription factor genes were identified from $M$. truncatula and a number of them were characterized by sequence alignment, northern hybridization analysis and transgenic overexpression. One of the genes, WXP1, has a unique function and was characterized in detail. The open reading frame (ORF) of WXP1 encodes a peptide of 371 aa with an estimated molecular mass of $41.3 \mathrm{kD}$. The deduced amino acid sequence of this gene contains one conserved AP2 domain. When aligned with well-characterized AP2 domain-containing transcription factors from Arabidopsis, tomato and tobacco, the AP2 domain of WXP1 shared high similarity to that of the other proteins.

Phylogenetic analysis showed that WXP1 is distinct from most of the known AP2 domain transcription factors based on analysis of their complete protein sequences. 
When compared with amino acid sequences of other stress or wax related genes, WXP1 is only $19.4-22.8 \%$ identical to AtCBFs, $16.7 \%$ to AtDREB2A, 20.6\% to AtDREB2B, 22.8-25.3\% to the three ERFs, $28.6 \%$ to AtWIN1 and 14.0\% to ZmGlossy15.

A large numbers of independent transgenic alfalfa plants were produced using the 35SWXP1 construct by Agrobacterium-mediated transformation. Transgenic lines $(18,41$, and 47) with different transgene expression levels (low, medium, high) were analyzed in detail.

Even though the overexpression of WXP1 did not result in severe growth retardation, the alfalfa transgenic lines tended to grow relatively slowly, particularly the high expressors. When compared with control plants, flowering time of the transgenic plants was delayed $5-28 \mathrm{~d}$, height of the transgenic lines were $2-32 \%$ shorter at flowering time, and trifoliates of the transgenic lines were smaller. Fresh and dry matter production of the transgenic lines decreased $5.6-26.8 \%$ and $6.7-42.7 \%$, respectively. The most obvious phenotypic change in the WXP1 overexpressed alfalfa plants was the more glaucous appearance in the leaves. The increased glaucousness occurred in most of the transgenic lines with low to extremely high WXP1 overexpression levels. Overexpression of WXP1 led to increased cuticular wax production and improved drought tolerance in transgenic alfalfa

Transgenic alfalfa lines and control plants were examined by scanning electron microscopy (SEM). Overexpression of WXP1 in alfalfa resulted in increased wax loading on both sides and earlier accumulation of epicuticular waxes on the adaxial surface. When the new leaf became fully expanded (top- $1^{\text {st }}$ ), there were no visible wax crystals on the adaxial leaf surface of control plants (Figure 1A); in contrast, wax crystal structures were already developed on the adaxial leaf surface of transgenic plants (Figures $1 \mathrm{~B}$ ). For the top- $2^{\text {nd }}$ trifoliate, transgenic plants had higher wax crystal density on the adaxial leaf surfaces than the control plants (Figures 1C and 1D). 

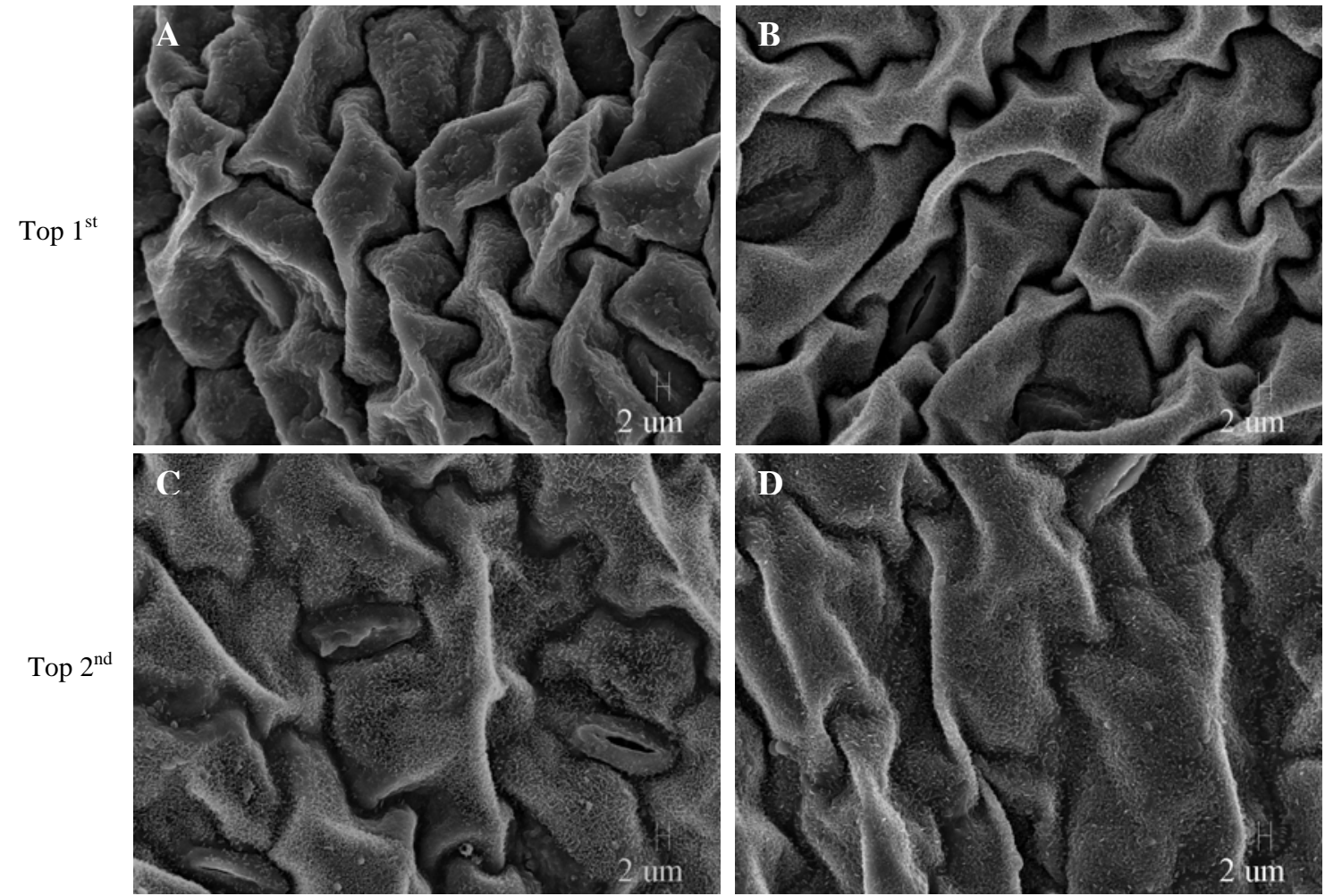

Figure 1. Epicuticular wax crystallization patterns on adaxial leaf surfaces of transgenic and control alfalfa plants viewed by scanning electron microscope. (A,C) empty vector control (VCTR). (B, D) Transgenic line 47. Images were taken at $\times 1,000$ magnification.

Total wax accumulation per leaf area was significantly higher in the transgenic lines than that in the control plants. The biggest change of cuticular wax deposition was in transgenic line 47, which had an increase of 37.7\% in its wax load. Lines 18 and 41 showed increases in their total wax load by $29.6 \%$ and $35.3 \%$, respectively. The increase was mainly contributed by C30 primary alcohol, which is the major component of alfalfa leaf wax. C30 alcohol in transgenic lines 18, 41 and 47 was 34.5\%, $43.0 \%$ and $45.3 \%$ more than that of the control plants, respectively. Other carbon length alcohols did not show significant changes.

Detached alfalfa trifoliates were subjected to leaf water loss rate assay. Both top$2^{\text {nd }}$ and top- $3^{\text {rd }}$ trifoliates from the transgenic lines showed lower water evaporation rate than that from the vector control.

Transgenic lines and control plants were subjected to drought stress in growth chambers and greenhouse. In the growth chamber experiments, 3 days after watering was stopped, all the control plants became wilted while all the transgenic plants still kept their whole plant turgor (Figure 2A). After one more week in the same chambers without watering, all the plants became dehydrated and dead-like. Upon resumption of the normal watering scheme, the transgenic plants recovered much faster and better than the control plants (Figure 2B). In the greenhouse experiments, it took longer time to completely dehydrate the plants because of the fluctuations in relative humidity. Nevertheless, after three cycles of drought stress-rewatering in a period of 4 weeks, most of the transgenic plants survived while the control plants failed to recover. 



Figure 2. Effects of drought stress on transgenic and control alfalfa plants. (A) Three days of no-watering in growth chamber. Control plants (left) wilted while transgenic plants (line 47, right) still look normal. (B) After 10 days of no-watering, normal watering scheme was resumed, and the dried plants started recovering. Transgenic plants (line 47, with orange label) recovered quicker than the controls (with white label).

\section{Discussion}

Sequence analysis revealed WXP1 is very different from other well-characterized transcription factors related to abiotic stress or wax accumulation, e.g. DREB/CBF from Arabidopsis (Jaglo-Ottosen et al. 1998, Liu et al. 1998), WIN1/SHN1 from Arabidopsis (Aharoni et al. 2004, Broun et al. 2004) and GL15 from maize (Moose and Sisco 1996). Functional characterization of WXP1 in transgenic alfalfa plants demonstrated its novelty.

Overexpression of transcription factor genes $D R E B / C B F$ in Arabidopsis activated a number of downstream genes that are involved in cold acclimation and drought adaptation. Although the transgenic Arabidopsis showed increased stress tolerance, no additional waxes were produced (Jaglo-Ottosen et al. 1998, Kasuga et al. 1999). Thus, the mechanism of $D R E B / C B F$ genes on drought tolerance improvement is different from that of WXP1. Overexpression of WXP1 activated wax production and led to the glaucousness appearance in leaves of transgenic alfalfa. The transgenic alfalfa plants showed reduced water loss and decreased epidermal permeability, therefore they were more drought tolerant than the control plants. Genetic and mutant studies have suggested that wax accumulation is a potential drought adaptation trait (Jefferson 1994); our study using isogenic lines (wild type control, empty vector control and transgenic plants) clearly demonstrates the positive effects of cuticular waxes on drought tolerance. Despite the fact that both drought tolerance and wax accumulation are complicated traits that are under the control of multiple genes, our results demonstrated that overexpression of a single transcription factor gene, $W X P 1$, could turn on wax production and improve plant drought tolerance.

Because growth of transgenic alfalfa plant was negatively affected when WXP1 was overexpressed under the control of the constitutive CaMV35S promoter, the use of epidermal-specific promoters to drive the expression of WXP1 is likely to solve the problem. This is a quick way to develop transgenic cultivars without other adverse 
effects. Although transgenic expression of WXP1 in alfalfa led to increase wax accumulation in leaves, it is not clear what the direct target genes of WXP1 are. The use of microarray technology may allow to identify the target genes of WXP1. It will also be interesting to isolate and characterize the WXP1 promoter in future studies.

\section{References}

Aharoni A, Dixit S, Jetter R, Thoenes E, van Arkel G, Pereira A (2004) The SHINE clade of AP2 domain transcription factors activates wax biosynthesis, alters cuticle properties, and confers drought tolerance when overexpressed inarabidopsis. Plant Cell 16, 2463-2480.

Broun P, Poindexter P, Osborne E, Jiang C-Z, Riechmann JL (2004) WIN1, a transcriptional activator of epidermal wax accumulation in Arabidopsis. Proceedings of the National Academy of Sciences of the USA 101, 4706-4711.

Cook DR (1999) Medicago truncatula - a model in the making! Current Opinion in Plant Biology 2, 301-304.

Gilmour SJ, Sebolt AM, Salazar MP, Everard JD, Thomashow MF (2000) Overexpression of the Arabidopsis CBF3 Transcriptional Activator Mimics Multiple Biochemical Changes Associated with Cold Acclimation. Plant Physiology 124, 1854-1865.

Haake V, Cook D, Riechmann JL, Pineda O, Thomashow MF, Zhang JZ (2002) Transcription Factor CBF4 Is a Regulator of Drought Adaptation in Arabidopsis. Plant Physiology 130, 639-648.

Hooker TS, Millar AA, Kunst L (2002) Significance of the Expression of the CER6 Condensing Enzyme for Cuticular Wax Production in Arabidopsis. Plant Physiology 129, 1568-1580.

Jaglo-Ottosen KR, Gilmour SJ, Zarka DG, Schabenberger O, Thomashow MF (1998) Arabidopsis CBF1 Overexpression Induces COR Genes and Enhances Freezing Tolerance. Science 280, 104-106.

Jefferson PG (1994) Genetic variation for epicuticular wax production in Altai wildrye populations that differ in glaucousness. Crop Science 34, 367-371.

Jenks MA, Ashworth EN (1999) Plant epicuticular waxes: function, production, and genetics. In 'Horticultural Reviews'. (Ed. J Janick) pp. 1-68. (John Wiley \& Sons, Inc.: New York, USA)

Jenks MA, Eigenbrode S, Lemeiux B (2002) Cuticular waxes of Arabidopsis. In 'The Arabidopsis Book'. (Eds C Somerville and E Meyerowitz) pp. doi: 10.1199/tab.0016. (American Society of Plant Biologists: Rockville, MD, USA)

Kasuga M, Liu Q, Miura S, Yamaguchi-Shinozaki K, Shinozaki K (1999) Improving plant drought, salt, and freezing tolerance by gene transfer of a single stressinducible transcription factor. Nature Biotechnology 17, 287 - 291.

Kunst L, Samuels AL (2003) Biosynthesis and secretion of plant cuticular wax. Progress in Lipid Research 42, 51-80.

Liu Q, Kasuga M, Sakuma Y, Abe H, Miura S, Yamaguchi-Shinozaki K, Shinozaki K (1998) Two Transcription Factors, DREB1 and DREB2, with an EREBP/AP2 DNA Binding Domain Separate Two Cellular Signal Transduction Pathways in Drought- and Low-Temperature-Responsive Gene Expression, Respectively, in Arabidopsis. Plant Cell 10, 1391-1406.

Millar AA, Clemens S, Zachgo S, Giblin EM, Taylor DC, Kunst L (1999) CUT1, an Arabidopsis Gene Required for Cuticular Wax Biosynthesis and Pollen Fertility, 
Encodes a Very-Long-Chain Fatty Acid Condensing Enzyme. Plant Cell 11, 825-838.

Moose S, Sisco P (1996) Glossy15, an APETALA2-like gene from maize that regulates leaf epidermal cell identity. Genes and Development 10, 3018-3027.

Naâ R, Markstaedter C, Hauke V, Riederer M (1998) Quantitative gas chromatographic analysis of plant cuticular waxes containing long-chain aldehydes.

Phytochemical Analysis 9, 112-118.

Novillo F, Alonso JM, Ecker JR, Salinas J (2004) CBF2/DREB1C is a negative regulator of CBF1/DREB1B and CBF3/DREB1A expression and plays a central role in stress tolerance in Arabidopsis. PROCEEDINGS OF THE NATIONAL ACADEMY OF SCIENCES OF THE USA 101, 3985-3990.

Okamuro JK, Caster B, Villarroel R, Van Montagu M, Jofuku KD (1997) The AP2 domain of APETALA2 defines a large new family of DNA binding proteins in Arabidopsis. Proceedings of the National Academy of Sciences of the USA 94, 7076-7081.

Rawson HM, Clarke JM (1988) Nocturnal transpiration in wheat. Australian Journal of Plant Physiology 15, 397-406.

Riechmann JL, Heard J, et al. (2000) Arabidopsis Transcription Factors: Genome-Wide Comparative Analysis Among Eukaryotes. Science 290, 2105-2110.

Shinozaki K, Yamaguchi-Shinozaki K, Seki M (2003) Regulatory Network of Gene Expression in the Drought and Cold Stress Responses. Current Opinion in Plant Biology 6, 410-417.

Stockinger EJ, Gilmour SJ, Thomashow MF (1997) Arabidopsis thaliana CBF1 encodes an AP2 domain-containing transcriptional activator that binds to the Crepeat/DRE, a cis-acting DNA regulatory element that stimulates transcription in response to low temperature and water deficit. Proceedings of the National Academy of Sciences of the USA 94, 1035-1040.

Young ND, Cannon SB, Sato S, Kim D, Cook DR, Town CD, Roe BA, Tabata S (2005a) Sequencing the Genespaces of Medicago truncatula and Lotus japonicus. Plant Physiology 137, 1174-1181.

Young ND, Cannon SB, Sato S, Kim DJ, Cook DR, Town CD, Roe BA, Tabata S (2005b) Sequencing the genespaces of Medicago truncatula and Lotus japonicus. Plant Physiology 137, 1174-1181.

Zhang J-Y, Broeckling CD, Blancaflor EB, Sledge M, Sumner LW, Wang Z-Y (2005) Overexpression of WXP1, a putative Medicago truncatula AP2 domaincontaining transcription factor gene, increases cuticular wax accumulation and enhances drought tolerance in transgenic alfalfa (Medicago sativa). Plant Journal 42, 689-707. 